Technische Universität IImenau Institut für Volkswirtschaftslehre

Diskussionspapier Nr. 93

Wettbewerb und Regulierung

Oliver Budzinski und Sandra Eckert

März 2015

Institut für Volkswirtschaftslehre

Ehrenbergstraße 29

Ernst-Abbe-Zentrum

D-98 684 Ilmenau

Phone 03677/69-4030/-4032

Fax 03677/69-4203

http://www.wirtschaft.tu-ilmenau.de

ISSN 0949-3859 


\title{
Wettbewerb und Regulierung
}

\author{
Oliver Budzinski* \& Sandra Eckert\#
}

(erscheint in: Karsten Mause, Christian Müller \& Klaus Schubert (Hrsg.), Politik und Wirtschaft: ein integratives Kompendium, Berlin: Springer 2015)

\begin{abstract}
Wettbewerb und Regulierung werfen sowohl aus einer wirtschafts- als auch aus einer politikwissenschaftlichen Perspektive interessante Fragestellungen auf und haben daher in beiden Disziplinen umfangreiche Beachtung gefunden. Der vorliegende Beitrag gibt eine Übersicht über beide Herangehensweisen. Dabei werden zunächst die grundlegenden Unterschiede und Gemeinsamkeiten offengelegt (Abschnitt 2), bevor die disziplinären Schwerpunkte in der Analyse vorgestellt, und aus Sicht der jeweils anderen Disziplin kommentiert werden (Abschnitte 3 und 4). Wir kommen zu dem Ergebnis, dass beide Sichtweisen in erster Linie komplementär sind und sich gegenseitig befruchten können.
\end{abstract}

Keywords: Wettbewerb, Regulierung, Wirtschaftswissenschaften, Politikwissenschaften, Wettbewerbspolitik

JEL-Codes: A12, A20, L40, L50, P40

Budzinski, Oliver, Prof. Dr., Leiter des Instituts für Volkswirtschaftslehre und des Fachgebietes Wirtschaftstheorie, Technische Universität IImenau, Fakultät für Wirtschaftswissenschaften und Medien, Ehrenbergstraße 29, D-98693 IImenau, oliver.budzinski@tu-ilmenau.de.

\# Eckert, Sandra, Prof. Dr., Juniorprofessur für Politik im Europäischen Mehrebenensystem, GoetheUniversität Frankfurt am Main, Fachbereich Gesellschaftswissenschaften, Institut für Politikwissenschaft, Theodor-W.-Adorno-Platz 6, 60323 Frankfurt am Main, Eckert@soz.uni-frankfurt.de. 


\section{Einleitung}

Wettbewerb und Regulierung aus einer interdisziplinären, nämlich sowohl wirtschafts- als auch politikwissenschaftlichen Perspektive zu betrachten, ist aus vielerlei Gründen ein gewinnbringender Ansatz. Vordergründig lohnt sich diese politökonomische Sichtweise vor allem deshalb, weil der Forschungsbeitrag der beiden Disziplinen genau spiegelverkehrt verortet ist: der Beitrag der Ökonomie liegt vor allem im Bereich Wettbewerb, der Beitrag der Politologie im Bereich Regulierung. Bei genauerem Hinsehen erweisen sich auch die unterschiedlich gelagerten Forschungsinteressen als komplementär: während die Wirtschaftswissenschaftlerinnen vor allem die unmittelbaren Wettbewerbseffekte in den Blick nehmen, interessieren sich die Politikwissenschaftler vor allem für die marktsteuernden Eingriffe, seien sie nun wettbewerbsbehördlicher oder regulierungsbehördlicher Natur. Diese Varianz bzgl. Forschungsbeitrag und -interesse machen wir uns zu Eigen, um unseren interdisziplinären Beitrag zu gliedern. Zunächst werden wir die Unterschiede der disziplinären Zugänge einführend aufzeigen (Unterkapitel 2), um dann sukzessive die ökonomische Domäne der Analyse von Wettbewerbswirkungen (Unterkapitel 3) sowie die politologische Domäne der Analyse regulativer Eingriffe (Unterkapitel 4) vorzustellen. Diese disziplinären Zugänge werden aus Sicht der jeweils anderen Disziplin kommentiert, und in den Schlussfolgerungen im Sinne einer transdisziplinären, politökonomischen Rezeption zusammen geführt.

\section{Disziplinäre Zugänge: Wettbewerb und Regulierung aus wirtschafts- und politikwissenschaftlicher Perspektive}

In der ökonomischen Disziplin herrscht eine klare Trennung zwischen Wettbewerbsökonomik und Regulierungsökonomik als Subdisziplinen der Wirtschaftswissenschaft. Hingegen werden wettbewerbsbehördliche und regulierungsbehördliche Eingriffe in der Politikwissenschaft weitgehend als Einheit, nämlich als Varianten der regulativen Politik, verstanden. 


\subsection{Wettbewerbsökonomik und Regulierungsökonomik als Subdisziplinen der Wirtschaftswissenschaft}

In den Wirtschaftswissenschaften werden sowohl die Wettbewerbsökonomik als auch die Regulierungsökonomik in der angewandten Mikroökonomik verortet. Traditionellerweise findet auch der Begriff der Industrieökonomik für die theoretischen Grundlagen beider Bereiche Anwendung, während der wirtschaftspolitische Bezug in einer Verwandtschaft zur politischen Ökonomik zum Ausdruck kommt. Das wettbewerbs- und regulierungsökonomische Denken setzt am Primat des Marktes als dezentraler Koordinationsmechanismus von Angebot und Nachfrage an. Dies geht auf den Nachweis der Unmöglichkeit einer zentralisierten Koordination individuellem Angebots- und Nachfrageverhalten (Hayek 1945, 1968) zurück, weswegen ein dezentraler Koordinationsmechanismus benötigt wird, um knappe Güter zu bewirtschaften. Die wirtschaftswissenschaftliche Literatur hat zudem herausgearbeitet, dass wettbewerbliche Märkte als dezentraler Koordinationsmechanismus eine Reihe von gesellschaftlichen Vorteilen aufweisen: sie steigern die Wohlfahrt (verstanden als Ausstattung der Volkswirtschaft mit knappen Gütern) durch eine effiziente Allokation (= Verteilung der Produktionsfaktoren auf die Anbieter), die Schaffung von Innovationsanreizen (dynamische Effizienz), der Förderung der Konsumentensouveränität (Anpassung der Güterproduktion an die Präferenzen der Nachfrager) und adaptive Effizienz (Anpassungsfähigkeit der Volkswirtschaft an exogene Schocks und Krisen). Zudem bedingen sich wirtschaftliche Freiheit und wettbewerbliche Märkte gegenseitig (Röpke 1949; Eucken 1952). Wird bejaht, dass Konsumenten frei entscheiden dürfen, was sie wann bei wem kaufen, dann entsteht zwangsläufig Wettbewerb zwischen Anbietern. Das Gleiche gilt, wenn Gewerbe- und Berufswahlfreiheit bejaht wird; auch dann entsteht zwangsläufig Konkurrenz zwischen denen, die Substitute (also Güter, die aus Sicht der Nachfrager gleiche oder ähnliche Bedürfnisse befriedigen) anbieten.

Dem entsprechend entsteht Raum für eine Wettbewerbs- und Regulierungspolitik aus ökonomischer Sicht immer dann, wenn es entweder zu Marktversagen kommt oder erhebliche Funktionsmängel von Märkten auftreten. Dabei werden Wettbewerbspolitik und Regulierungspolitik üblicherweise als zwei unterschiedliche Felder 
verstanden. Dies liegt daran, dass Marktwettbewerb nach dominierender ökonomischer Auffassung nur dann nachhaltig funktioniert, wenn er gegen inhärente Selbstauflösungstendenzen geschützt wird (Vanberg 1999, 2000). Die im Wettbewerb stehenden Unternehmen können einen betriebswirtschaftlichen Erfolg stets entweder durch Leistung im Wettbewerb (bspw. bessere Produkte, besserer Service, bessere Preis-Leistungs-Verhältnisse, bessere Innovationen, etc.) oder durch Ausschaltung des Wettbewerbs (Kartellierung, nicht-leistungsgerechte Monopolisierung, Missbrauch von Marktmacht, siehe Abschnitt 3.1.1) erzielen - denn in letzterem Fall können die unternehmerischen Gewinne zu Lasten der Konsumenten und der Gesellschaft gesteigert werden. Daher bedarf es einer Wettbewerbspolitik, die den Wettbewerbsprozess schützt und die Ausschaltung des Wettbewerbes durch die Marktteilnehmer (sog. private Wettbewerbsbeschränkungen) verhindert.

Dem gegenüber spricht man in der Ökonomik von Regulierungspolitik, wenn der marktliche Wettbewerbsprozess aufgrund anderer Faktoren gestört ist und die Politik versucht, diese Dysfunktionalitäten zu korrigieren (siehe Abschnitt 3.1.2). Marktversagen liegt vor (Fritsch 2014), wenn Güter von so starken positiven Externalitäten gekennzeichnet sind, dass sie den Charakter von öffentlichen Gütern aufweisen (Gleichzeitigkeit von Nicht-Rivalität im Konsum und Nicht-Ausschließbarkeit von Trittbrettfahrern), oder Kostenstrukturen so stark von Subadditivität gekennzeichnet sind, dass selbst die gesamte Marktmenge von nur einem Unternehmen zu den niedrigsten Grenzkosten bereitgestellt werden kann (natürliche Monopole). In diesen seltenen Fällen kommen wettbewerbliche Märkte aufgrund der speziellen Güter- oder Produktionscharakteristika nicht zustande. Funktionsstörungen können bei sonstigen (technologischen) Externalitäten und bei erheblich und persistent asymmetrischen Informationen zwischen Anbietern und Nachfragern vorliegen. ${ }^{1}$ Darüber hinaus regulieren reale Staaten typischerweise weitere Märkte, welche eigentlich keine Funktionsstörungen aufweisen (sog. staatliche Wettbewerbsbe-

\footnotetext{
Es ist zu betonen, dass Marktversagen und Funktionsstörungen lediglich bedeuten, dass Spielraum für wohlfahrtssteigernde Regulierungen besteht. Damit ist nicht gesagt, dass reale Regulierung auch zu einer Wohlfahrtssteigerung führt. Vielmehr kommt es (nicht selten) vor, dass das Ergebnis der Regulierung noch schlechter ist als jenes des funktionsgestörten oder ,versagenden' Marktes.
} 
schränkungen). Gründe hierfür können in übergeordneten gesellschaftlichen Zielen liegen, aber auch in erfolgreichem Lobbyismus bestimmter gut organisierter Interessengruppen und in Imperfektionen des politischen Prozesses.

Sowohl in der Wettbewerbs- als auch in der Regulierungsökonomik steht traditionell die Wirkungsanalyse im Mittelpunkt, das heißt die Frage, wie wirken sich Beschränkungen des Wettbewerbs durch private Akteure (sog. private Wettbewerbsbeschränkungen, siehe Abschnitt 3.1.1) bzw. wie wirken sich regulatorische Eingriffe des Staates in die Marktprozesse auf die Wohlfahrt aus. Bezüglich des Verständnisses von Wohlfahrt dominieren dabei zwei Konzepte: zum einen die Gesamtwohlfahrt und zum anderen die Konsumentenwohlfahrt. Wesentlicher Unterschied ist die Berücksichtigung von Umverteilungseffekten zwischen Produzenten und Konsumenten: während es für das Gesamtwohlfahrtskonzept unerheblich ist, bei wem Wohlfahrt anfällt und mithin reine Umverteilungen von den Konsumenten zu den Produzenten nicht als Wohlfahrtsverlust gewertet werden, legt der Konsumentenwohlfahrtsstandard mehr Gewicht auf die den Konsumenten zufallende Wohlfahrt und wertet Umverteilungen zu Lasten der Konsumenten und zu Gunsten der Produzenten als wohlfahrtsschädlich. Es gibt in der Wirtschaftswissenschaft keinen Konsens darüber, welches Wohlfahrtskonzept vorzuziehen ist. ${ }^{2}$

\subsection{Regulative Politik als Einheit in den Politikwissenschaften}

Wie eingangs erwähnt liegt der politikwissenschaftliche Beitrag nicht in der Analyse von Wettbewerbseffekten, sondern in der Untersuchung nachgelagerter marktsteuernder Eingriffe. Normativ gesehen ist eine politikwissenschaftliche Perspektive regulierungsfreundlicher als eine ökonomische Perspektive, zumal die Modellierung der Funktionsweise von Märkten und Wettbewerb nicht Gegenstand der Analyse ist. Aus Sicht der Ökonomik interessiert sich die Politikwissenschaft prioritär für die Regulierungspolitik, und hierbei für Eingriffe, die nicht direkt durch Dysfunktionalitäten begründet sind. Im Zentrum steht die Realisierung übergeordneter gesellschaftlicher, also gemeinwohlorientierter Ziele, wobei das politikwissenschaftliche

Vgl. zu verschiedenen Standpunkten in einer jüngeren Kontroverse, welche auch das Freiheitsziel einschließt n.v.a Hellwig (2006), Weizsäcker (2007), Budzinski (2008b) und Vanberg (2011). 
Wohlfahrtskonzept umverteilenden Implikationen Rechnung trägt. Dies heißt nicht, dass die Politikwissenschaft das Feld der Wettbewerbspolitik im Sinne der Wettbewerbsökonomik völlig außer Acht lässt. Vielmehr werden Maßnahmen, die auf den Schutz des Wettbewerbes abzielen, nicht gesondert betrachtet, sondern als mit anderen Zielsetzungen konkurrierend. Wettbewerbsfördernde Maßnahmen sind nur eine mögliche Variante einer regulativen Politik, die im Gegensatz zur Ökonomie als Einheit betrachtet wird.

Einflussreich für eine solche einheitliche Sichtweise auf Wettbewerbspolitik und Regulierung war insbesondere Lowis (1972) typologische Unterscheidung zwischen vier Politikarenen je nachdem, ob es sich um verteilende, umverteilende, regulative oder konstitutive Politiken handelt (besprochen bei Heinelt 2003). Gemäß Lowis Hauptthese "policy determines politics" unterscheiden sich diese vier Arenen systematisch bezüglich ihres Konfliktpotentials. Dieser zufolge zeichnet sich vor allem die redistributive Politik aufgrund der Sichtbarkeit ihres umverteilenden Charakters durch ein hohes Maß an Konfliktpotential aus, während die distributive Politik durch bloße Zuteilung von Ressourcen bereits weniger konfliktträchtig sei, das geringste $\mathrm{Ma}$ an Konfliktpotential aber bei regulativer Politik vorläge. Obwohl die Implikationen dieser These durchaus kontrovers diskutiert wurden, und insbesondere der indirekt umverteilende Charakter regulativer Politik thematisiert wurde, blieb vor allem die Unterscheidung zwischen verteilenden und umverteilenden Politiken einerseits, und regulativen Politiken andererseits, wirkungsmächtig. So definiert beispielsweise Adrienne Héritier in ihrem für die deutsche Policyforschung wegweisenden Beitrag (Héritier 1987: 39) regulative Politik als verhaltensnormierende Politik ohne Leistungscharakter. Unter diese Kategorie fallen neben regulierungspolitischen auch wettbewerbspolitische Eingriffe, beispielsweise kartellrechtlicher Natur (Héritier 1987: 40). Die Unterscheidung regulativer versus nicht bloß regelsetzender, weil verteilender oder umverteilender Politik, setzt sich fort in zentralen Begriffen wie regulativer Staat (Majone 1994), regulative Governance (Levi-Faur 2007; Eckert 2011) oder regulativer Kapitalismus (Levi-Faur \& Jordana 2005; Braithwaite 2008). 
Wettbewerb und Regulierung werden in den Politikwissenschaften also als Varianten der regulativen Politik gemeinsam erfasst, und eine weitere Ausdifferenzierung erfolgt gemäß der Zielsetzung von Markteingriffen. So unterschied beispielsweise Héritier zwischen kompetitiv-regulativen, protektiv-regulativen und sozialregulativen Policies (Héritier 1987: 157, 174, 176). Kartellrechtliche Eingriffe sind so dem Bereich der kompetitiv-regulativen Policy zuzuordnen, die „das Marktverhalten und Marktzutrittsverhalten von privaten Unternehmen“ regeln sollen. Weniger differenziert ist die Unterscheidung zwischen ökonomischer und sozialer Regulierung, die in der politikwissenschaftlichen Literatur aufzufinden ist (z. B. Czada \& Lütz 2003). Diese konzeptuell recht unscharfe Unterscheidung entspricht noch am ehesten der Unterscheidung zwischen den Subdisziplinen Wettbewerbs- und Regulierungsökonomik, denn unter ökonomischer Regulierung werden in der Regel wettbewerbsfördernde Maßnahmen erfasst.

Der wesentliche Unterschied zur Ökonomie besteht aber darin, dass nicht die Wirkungen dieser politischen Eingriffe im Zentrum stehen, sondern vielmehr die institutionellen und prozeduralen Rahmenbedingungen, innerhalb derer sie stattfinden, sowie die zugrunde liegenden Motivationen. Aus institutioneller Sicht ist dabei von Interesse, dass sogenannte nicht majoritäre Institutionen (Thatcher \& Stone Sweet 2002), die nicht politisch legitimiert sind und in einem beschränkten Kompetenzbereich über Befugnisse verfügen, ein Charakteristikum der regulativen Politik, nicht aber der verteilenden und umverteilenden Politik sind. Die Grenzen zwischen Wettbewerbs- und Regulierungspolitik hingegen sind aus politikwissenschaftlicher Sicht sowohl prozedural, institutionell wie auch substantiell fließend: wie die Regulierungspolitik operiert Wettbewerbspolitik innerhalb eines rechtlichen Rahmens, der ex ante Regeln setzt und verhaltensnormierend wirkt. In beiden Bereichen spielen nichtgewählte Institutionen eine zentrale Rolle, seien dies unabhängige Wettbewerbsbehörden, oder Regulierungsbehörden. Und in der Regel überschneidet sich der Zuständigkeitsbereich sektorspezifisch agierender Regulierungsagenturen mit demjenigen von Wettbewerbsbehörden im jeweils regulierten Sektor, bzw. es liegt zumindest eine Notwendigkeit der Koordination und Kooperation vor. Viele Regulierungsbehörden sind gleichzeitig mit der Umsetzung wettbewerbsrechtlicher Vor- 
gaben betraut, während Wettbewerbsbehörden über Zeit sektorspezifische Expertise in zentralen Wirtschaftsbereichen wie etwa Energiemärkte entwickeln, und diese in eigenständigen Referaten und Regularien verstetigen. Substantiell liegt die Überschneidung dort, wo durch Regulierungs- und Wettbewerbsbehörden einerseits wettbewerbsfördernde Maßnahmen ergriffen werden, andererseits berechtigte Ausnahmen von Marktprinzipien definiert und durchgesetzt werden.

\section{Die ökonomische Domäne: Die Analyse von Wettbewerbswirkungen von} Unternehmensstrategien und staatlichen Interventionen

\subsection{Die ökonomische Wirkungsanalyse: Methoden und zentrale Ergebnisse}

\subsubsection{Prowettbewerbliche und antiwettbewerbliche Unternehmensstrategien}

Die wirtschaftswissenschaftliche Analyse der Wettbewerbspolitik konzentriert sich auf die Wirkungen unternehmerischer Strategien und Arrangements auf den Wettbewerb (siehe auch Abschnitt 2.1). Dabei steht der Versuch im Mittelpunkt, eine möglichst genaue Abgrenzung zwischen wettbewerbskonformen und wettbewerbswidrigen Strategien und Arrangements zu finden, um der Wettbewerbspolitik mit Hilfe der ökonomischen Analyse zu ermöglichen, Wettbewerbsbeschränkungen möglichst fehlerfrei zu identifizieren und mit Hilfe des Wettbewerbsrechtes zu bekämpfen. Dabei kann die ökonomische Wirkungsanalyse sowohl dazu verwendet werden, wohlfahrtsoptimale Wettbewerbsregeln (inklusive ihrer Auslegung) zu formulieren (regelbasierte Wettbewerbspolitik; bspw. Budzinski 2010, 2013c) als auch dazu, die pro- oder antiwettbewerblichen Effekte konkreter Fälle zu analysieren und abzuwägen (evidenzbasierte Wettbewerbspolitik; bspw. Röller 2005; Neven 2006). In der Wettbewerbsökonomik unterscheidet man typischerweise zwischen drei Typen potenziell wettbewerbswidriger Strategien und Arrangements der Marktteilnehmer (bspw. Motta 2004; Van den Bergh \& Camesasca 2006; Kerber 2007; Kerber \& Schwalbe 2007; Bishop \& Walker 2010; Haucap \& Schmidt 2013; Blair \& Sokol 2015): (a) Kartelle und Kartellsurrogate, (b) Missbrauch von Marktmacht und (c) Unternehmenszusammenschlüsse und Konzentration. Zwei weitere Bereiche werden teilweise hinzugezählt, nämlich (d) unfaires Wettbewerbsverhalten 
und (e) staatliche Beihilfen und Subventionen (Schmidt \& Schmidt 2006; Buccirossi 2008). ${ }^{3}$

(a) Kartelle und Kartellsurrogate (Choi \& Gerlach 2015; Levenstein \& Suslow 2015)

Immer dann, wenn mindestens ein Wettbewerbsparameter zwischen mindestens zwei Unternehmen durch kooperative Koordination (Absprachen) festgelegt und damit der Konkurrenz zwischen den Unternehmen entzogen wird, spricht man von Kollusion. Erfolgt diese auf vertraglicher Basis, so handelt es sich um ein Kartell. Liegt der Kollusion kein Vertrag zu Grunde spricht man von einem Kartellsurrogat oder kollusivem Parallelverhalten. Während diese Unterscheidung aus rechtswissenschaftlicher Sicht von erheblicher Bedeutung ist (u.a. in Bezug auf Beweisanforderungen), so wirken Kartelle und Kartellsurrogate aus ökonomischer Sicht grundsätzlich gleich (zu diesem Gegensatz Franck 2015), so dass diese Unterscheidung für die Wirkungsanalyse nachfolgend vernachlässigt und stellvertretend nur von Kartellen ausgegangen werden kann.

Ein wesentlicher Kartelltyp ist das sogenannte horizontale Preiskartell, bei welchem der Wettbewerbsparameter Preis zwischen Unternehmen abgesprochen wird, die direkt im Wettbewerb zueinander stehen. Dies ist aus unternehmerischer Sicht nur dann profitabel (und damit rational), wenn entweder alle Unternehmen des Marktes oder zumindest der allergrößte Teil beteiligt sind, denn andernfalls werden die Nachfrager größtenteils zu jenen Wettbewerbern abwandern, welche mit dem Kartell über den Preis konkurrieren, mithin ein günstigeres Preis-Leistungs-Verhältnis anbieten. Ist das Preiskartell hingegen marktumfassend, so können sich die kartellierten Unternehmen wie ein Monopolist verhalten und gemeinsam den Preis auf

Motta (2004), Schmidt \& Schmidt (2006), Van den Bergh \& Camesasca (2006), Kerber (2007), Kerber \& Schwalbe (2007), Buccirossi (2008), Bishop \& Walker (2010), Haucap \& Schmidt (2013) sowie Blair \& Sokol (2015) enthalten alle ausführliche Kapitel zu den nachfolgend sehr kurz skizzierten Arten von Wettbewerbsbeschränkungen (Theorie) sowie auch zu den nachfolgend kurz thematisierten Methoden ihrer Messung (Empirie). Im Weiteren wird daher nur stellenweise auf zusätzliche Literatur verwiesen, wobei wir uns auf Übersichtartikel und Handbuchbeiträge beschränken. Die sehr umfangreiche Primärliteratur würde den Rahmen dieses Beitrages sprengen und lässt sich anhand der dort zitierten Literatur nachvollziehen. 
den Monopolpreis heben und den überwettbewerblichen Gewinn unter sich verteilen. Dies ist generell wohlfahrtsschädlich, da der Wettbewerb nun zum Erliegen kommt (also die statischen und dynamischen Wettbewerbsfunktionen nicht mehr gegeben sind; siehe 2.1) und die Gewinne der Kartellanten zu Lasten der Nachfrager und der Gesellschaft gehen, wobei die Verluste der Letztgenannten die unternehmerischen Zusatzgewinne überkompensieren. Neben einem vollständig marktumfassenden Preiskartell sind auch solche Preiskartelle denkbar, welche alle Anbieter beinhalten, welche über relevante Marktanteile und Kapazitätsreserven bzw. kurzfristige Wachstumspotenziale verfügen. Die verbleibende Restkonkurrenz kann dann nicht mehr als einen geringen Bruchteil des Marktes bedienen, so dass sich das Kartell gegenüber dem größten Teil der Nachfrager wiederum als Monopolist verhalten kann, ohne dass es signifikante Abwanderung der Nachfrager zu den Kartellaußenseitern befürchten muss. Oftmals wird es dann so sein, dass sich die verbleibenden Kartellaußenseiter in ihrer Preispolitik dem Kartell anpassen, da sie durch attraktivere Preis-Leistungs-Angebote mangels möglicher Kapazitätsausweitung keine Profitverbesserung erzielen können. Sie schlüpfen dann sozusagen unter den „Schutzschirm” des Kartells (umbrella effects; Inderst et al. 2014).

Preiskartelle werden also von rationalen und gewinnmaximierenden Unternehmen generell nur dann eingegangen werden, wenn sie den Wettbewerb auf dem relevanten Markt vollständig ausschalten und die wettbewerbliche Koordination vollständig durch eine kollusive Koordination ersetzen. Mithin sind Preiskartelle aus ökonomischer Sicht immer wohlfahrtsschädlich. Dieselbe Logik gilt auch für Mengenkartelle (Absprache der absoluten Angebotsmengen), Quotenkartelle (Absprache der Anteile der Kartellanten an einer definierten Gesamtmarktmenge) sowie Marktaufteilungskartellen (Festlegung von Gebietsmonopolen oder Absprache exklusiver Produktkategorien für jeden Kartellanten), weswegen alle diese Kartelltypen auch als Hardcorekartelle bezeichnet werden.

Auch hinsichtlich aller weiteren Parameter, über die Unternehmen Wettbewerb treiben können, können sich Kartelle bilden, beispielsweise (jeweils horizontale) Innovationskartelle, Beschaffungs- und Vertriebskartelle, Standardisierungskartelle, 
Konditionenkartelle usw. Anders als bei den Hardcorekartellen können hier allerdings kaum generelle Aussagen über die Wettbewerbs- und Wohlfahrtswirkungen gemacht werden. Vielmehr hängen die Wettbewerbswirkungen typischerweise sensitiv von der Ausgestaltung im Einzelfall ab und unternehmerische Vorteile können meist auch bereits erzielt werden, wenn diese Kartelle nur wenige der relevanten Wettbewerber umfassen (und somit signifikanter Wettbewerb hinsichtlich dieser Parameter erhalten bleibt). Beispielsweise können Innovationskartelle einerseits wohlfahrtsfördernd wirken, wenn erst die kooperative Bündelung der (komplementären) Kompetenzen mehrerer Unternehmen die Entwicklung eines neuen Produktes oder einer neuen Technologie ermöglicht (bzw. die Kosten der Innovation senkt). Andererseits kann sich durch Innovationskartelle auch die Geschwindigkeit der Innovation verringern, weil Anreize durch den Wettbewerb um innovative Lösungen verloren gehen, und umfassende Innovationskooperationen verringern typischerweise die Anzahl der Wege, auf denen parallel nach besseren Produkten und Technologien gesucht wird, ohne dass ex ante bekannt ist, welche Wege mittelfristig die erfolgversprechenden wären (Verringerung der fruchtbaren Diversität). Als zweites Beispiel für die ambivalenten Effekte anderer Kartellarten sei auf Standardisierungskartelle verwiesen. Diese können den Wettbewerb sogar intensivieren und damit die Wohlfahrt erhöhen, wenn durch die Standardisierung von Verbindungen (Stecker, Kabel, Adapter, etc.) die Kompatibilität zwischen unterschiedlichen Anbietern erhöht wird (bspw. bei Ladegeräten für mobile Telefone und Computer). Werden freilich ganze Produkte standardisiert, so dass es keine Auswahl mehr für die Nachfrager gibt, kann der Wettbewerb auch signifikant eingeschränkt werden.

Neben den bisher diskutierten horizontalen Kartellen, also Absprachen zwischen Unternehmen, die in demselben Markt agieren, treten auch vertikale Kartelle auf. Hierbei treffen Unternehmen Absprachen, welche zwar entlang der gleichen Wertschöpfungskette tätig sind, aber auf verschiedenen Stufen, die einander vor- oder nachgelagert sind, d.h. die Kartellanten stehen zueinander in einer ZuliefererAbnehmer-Beziehung. Hier kann es insbesondere dann zu negativen Wettbewerbswirkungen kommen, wenn auf einzelnen Stufen der Wertschöpfungskette Marktmacht vorliegt (im Extremfall: wesentliche Einrichtungen oder natürliche Monopo- 
le), denn dann stellen vertikale Kartelle ein Instrument dar, um diese Marktmacht entlang der Wertschöpfungskette zu übertragen und Konkurrenten auf vor- oder nachgelagerten Stufen zu verdrängen - mit negativen Wirkungen insbesondere auf die dynamischen Wettbewerbsfunktionen.

\section{(b) Missbrauch von Marktmacht (White 2015)}

Erlangt ein einzelnes Unternehmen auf einem Markt ein Monopol oder eine marktbeherrschende Stellung (auch: Quasimonopol), so kann es diese Stellung beziehungsweise die daraus resultierende Finanz- und Lobbykraft missbrauchen, um den Wettbewerb zum Schaden der gesellschaftlichen Wohlfahrt dauerhaft auszuschalten, indem es bspw. keine neuen Wettbewerber in den Markt lässt (Abschottungsstrategien: Marktzutrittsschranken), verbleibende Restkonkurrenz aus dem Markt drängt (Verdrängungsstrategien: predatory pricing, Preisdiskriminierung, bundling \& tying, Verschlussstrategien, Boykotte usw.; Tirole 2005, Elzinga \& Mills 2015), die verbleibende Restkonkurrenz künstlich behindert (Behinderungsstrategien: raisingrivals' costs; Scheffman \& Higgins 2015), sich regulatorische oder politische Privilegien verschafft oder seine Marktmacht auf andere Märkte überträgt (leveraging) auch ohne die Existenz eigener Leistungsvorteile auf diesen Märkten. Zudem kann das marktbeherrschende Unternehmen Zulieferer, Abnehmer und Kunden ausbeuten, indem es überhöhte Preise verlangt (bzw. die Mengen künstlich einschränkt, was denselben Effekt hat) bzw. zu niedrige Zulieferpreise abpresst (Orbach \& Avraham 2015).

Vor dem Hintergrund der Vielfältigkeit der Missbrauchsmöglichkeiten von Monopolpositionen und marktbeherrschenden Stellungen könnte gefragt werden, ob die reine Existenz solcher Marktmacht nicht bereits wohlfahrtsschädlich ist - und damit wettbewerbspolitisch zu verhindern sei. Dabei ist allerdings zu beachten, dass manche erwünschten, wohlfahrtssteigernden Unternehmensstrategien unter bestimmten Umständen in marktbeherrschende Stellungen (und selten auch in Monopole) münden können. Tiefgreifende Innovationen neuer Produkte oder Technologien können beispielsweise Märkte komplett umkrempeln bzw. sogar neue Märkte schaffen und damit zwangsläufig zu dominierenden Stellungen der Innovatoren 
führen (Innovationsmonopole). Zudem kann es zu marktbeherrschenden Stellungen kommen, wenn ein Unternehmen besser und effizienter arbeitet als seine Konkurrenten (Leistungsmonopole). Beide Phänomene weisen daraufhin, dass die Marktstruktur letztendlich dem Wettbewerbsprozess endogen ist: kreative (innovationsund leistungsmonopolisierende) und imitierende (polypolisierende) Wettbewerbskräfte liefern sich einen dauerhaften, permanenten und evolutorischen Prozess der Vorstoßens und Aufholens (Clark 1961; Budzinski 2008a: 308-313). Damit wird aber auch klar, dass solche Innovations- und Leistungsmonopole meist temporärer Natur sind und durch den dynamischen Wettbewerb erodiert werden - es sei denn, dies wird durch einen Missbrauch der marktbeherrschenden Stellung zur Beschränkung der Wettbewerbskräfte seitens des dominanten Unternehmens unterbunden oder verzögert. Insofern ist aus ökonomischer Sicht nicht die innovations- oder leistungsbedingte Entstehung von Marktmacht das Problem, sondern ,lediglich' der nicht-wettbewerbliche Missbrauch dieser Macht.

\section{(c) Unternehmenszusammenschlüsse (Schwalbe \& Zimmer 2011)}

Das Entstehen oder Verstärken von Marktmacht ist allerdings dann als wettbewerbs- und wohlfahrtsschädlich einzuschätzen, wenn es nicht infolge von Innovations- oder Leistungswettbewerb auftritt, sondern durch Zusammenschlüsse von Unternehmen hergestellt wird, die bisher im Wettbewerb zueinander standen (horizontale Unternehmenszusammenschlüsse). Dies ist vergleichsweise offensichtlich, wenn durch Zusammenschlüsse (unabhängig davon, ob es sich um Fusionen bzw. Verschmelzungen, die Übernahme von Mehrheitsbeteiligungen oder die Übernahme solcher Minderheitsbeteiligungen handelt, die dennoch einen wesentlichen Einfluss auf die Unternehmensstrategie erlauben) ein Monopol oder eine marktbeherrschende Stellung entsteht oder letztere verstärkt wird. In der modernen Ökonomik kennt man allerdings noch zwei weitere Fälle wettbewerbswidriger horizontaler Zusammenschlüsse. Entstehen oder verstärken sich im Zuge oligopolistischer Zusammenschlüsse sogenannte koordinierte Effekte (Jayaratne \& Ordover 2015), dann führt der betreffende Zusammenschluss zu einem wesentlichen Absinken der Wettbewerbsintensität, weil sich die nach der Fusion verbleibenden Unternehmen keine oder nur noch deutlich reduzierte Konkurrenz liefern (Dominanz konsolidie- 
render Unternehmensstrategien; "Schlafmützenwettbewerb"). Dies geschieht vorzugsweisen in relativ homogenen Märkten mit einem engen Oligopol, in welchem die wettbewerbliche Aktivität vor allem von einem Unternehmen (dem sogenannten maverick) ausgeht, welches durch die Übernahme dann sozusagen ruhiggestellt wird. Hingegen entstehen unilaterale Oligopoleffekte (Keating \& Willig 2015) vorzugsweise in heterogenen Oligopolen, wenn dort jene Unternehmen fusionieren, deren Produkte besonders enge Substitute sind, also die dieselbe Marktnische besetzen bzw. aus Sicht der Nachfrager die engsten Konkurrenten darstellen, und dem Rückgang der Wettbewerbsintensität keine überkompensierenden fusionsbedingten Effizienzgewinne gegenüberstehen.

Vertikale und konglomerate Unternehmenszusammenschlüsse werden in der Ökonomik typischerweise als weniger gefährlich für den Wettbewerb eingeschätzt als horizontale Zusammenschlüsse. Allerdings können auch sie zu negativen Wettbewerbswirkungen führen, bspw. wenn vertikale Integration dazu führt, dass gesamte Wertschöpfungsketten dem Wettbewerb entzogen werden oder entstehende Konglomerate ihre Finanz- und Lobbykraft missbrauchen bzw. über (u.a.) die Ausnutzung von Komplementaritäten Wettbewerbsbeschränkungen auf zusätzliche Märkte ausweiten (Salinger 2015).

\section{(d) Unfaires Wettbewerbsverhalten}

Auch wenn sich die Wirtschaftswissenschaft damit vergleichsweise wenig beschäftigt, so besteht doch weitgehend Einigkeit darüber, dass bestimmte Formen des Behinderungswettbewerbs (bspw. unwahre oder irreführende Angaben, Anschwärzung und Verleumdung, Industriespionage und Sabotage, etc.) auch ohne die Existenz von Marktmacht auftreten können und dann negative Wirkungen entfalten.

\section{(e) Staatliche Beihilfen und Subventionen}

Wenn der Staat selektiv in funktionierende Märkte (Abwesenheit von Marktversagen; siehe Abschnitt 2.1.) eingreift und einzelne Wettbewerber subventioniert und andere nicht (oder Wettbewerber ungleichmäßig mit Subventionen bedenkt), dann verzerren diese Eingriffe den Wettbewerb mit typischerweise (spätestens mittelfris- 
tig) wettbewerbsmindernden Effekten und negativen Wohlfahrtswirkungen. Dabei ist es aus ökonomischer Sicht unerheblich, ob die Subvention oder Beihilfe direkter monetärer Natur ist oder indirekt monetäre Vorteile (bspw. Steuererleichterungen) bzw. nicht-monetäre Vorteile gewährt werden.

\subsubsection{Methoden und Relevanz}

Diese Kurzübersicht verdeutlicht, dass die Frage der ökonomischen Wirkungen potenziell wettbewerbsschädlicher Verhaltensweisen neben einem ausgefeilten theoretischen Fundament auch eine Frage der empirischen Analyse ist, also der Messung der tatsächlichen Effekte konkreter Fälle - sei es um die Wirkungen dieses Falles abzuschätzen oder um aus den Wirkungen typischer Fälle für geeignete wettbewerbsrechtliche Regeln und wettbewerbspolitische Praktiken zu lernen. Die Ermittlung konkreter Effekte einzelner Fälle ist dabei sowohl in die Vergangenheit gerichtet (forensische Ökonomik; Ewald 2011) als auch auf die Prognose zukünftiger Effekte (Budzinski 2010). Eine vergangenheitsorientierte Analyse wird bspw. benötigt, wenn es um die Aufdeckung illegaler Kartelle und die Ermittlung der durch sie verursachten Schäden (Doose 2014) geht, oder wenn vergangene Entscheidungen einer Wettbewerbsbehörde im Sinne einer Ex post-Evaluation überprüft werden (Budzinski 2012, 2013a). Geht es hingegen um die wettbewerbspolitische Beurteilung eines anstehenden Zusammenschlussvorhabens oder um die Abschätzung der Wirkungen geplanten Auflagen oder Zusagen, so stehen die zu erwartenden, zukünftigen Effekte, welche dieser Zusammenschluss in den betroffenen Märkten voraussichtlich haben wird, im Mittelpunkt der ökonomischen Analyse. Im Zuge der empirischen Analyse werden gängige quantitative Verfahren der Ökonometrie (bspw. Korrelationsanalysen, Difference-in-Difference Verfahren oder auch Eventstudien; Rubinfeld 2010) ebenso angewendet wie ausgefeilte und aufwendig kalibrierte Simulationsmodelle (Budzinski \& Ruhmer 2010). Hinzu kommen qualitative Methoden wie Dokumentenanalysen, Surveys (Konsumentenbefragungen oder solche beteiligten bzw. betroffenen Unternehmen), Experteneinschätzungen usw.

Die moderne Wettbewerbs- und Industrieökonomik ist der Analyse der wettbewerblichen Wirkungen von Unternehmensstrategien sehr weit fortgeschritten und 
beeinflusst zum einen die Ausgestaltung wettbewerbsrechtlicher Regeln und wettbewerbspolitischer Vorgehensweisen und informiert zum anderen die wettbewerbsrechtliche Handhabung konkreter Fälle in erheblicher Weise. Dennoch ist zu betonen, dass insbesondere der quantitativen Analyse von Einzelfällen auch weiterhin Grenzen gesetzt sind. Dies gilt zum einen im Hinblick auf den Stand der Theorie und der verfügbaren Methoden (Budzinski 2011) als auch in Bezug auf die Passung von ökonomischem Instrumentarium und rechtlichen Verfahrensanforderungen (Budzinski 2010; Christiansen 2010). Eine natürliche Grenze ist der Wirkungsanalyse immer dann gesetzt, wenn es um die Prognose zukünftiger Effekte geht, die niemals exakt und/oder unbedingt sein kann.

\subsection{Politikwissenschaftlicher Kommentar}

Trotz der internationalen Diffusion wettbewerbspolitischer Regime und Behörden hat die Politikwissenschaft diesem Politikfeld vergleichsweise wenig Aufmerksamkeit gewidmet, so dass das Gros der Publikationen im Bereich der Wirtschafts- und Rechtswissenschaften liegt. Seit den 1990er Jahren haben sich einzelne Beiträge, die in der Vergleichenden Politikwissenschaft sowie der politischen Ökonomie zu verorten sind, mit diversen Wettbewerbsregimen (Doern et al. 1996), sowie insbesondere mit der expandierenden Europäischen Wettbewerbspolitik (Cini \& McGowan 2008; Aydin \& Thomas 2012) befasst. Die Europäisierung der Wettbewerbspolitik ist vor allem aus einer Governanceperspektive von Interesse, da sie Fragen bezüglich der Kompetenzverlagerung im Europäischen Mehrebenenkontext aufwirft (Töller 2008). Im Kontext der disziplinären Debatte um den Aufstieg eines regulativen Staates (Majone 1994) wurde der Wettbewerbspolitik zentrale Bedeutung als prototypischer regulativer Politik beigemessen, die ohne nennenswerte finanzielle Ressourcen etabliert werden kann, und deren Kosten dezentral von Gerichten und den Rechtssystem sowie von Privatakteuren getragen werden. Das politikwissenschaftliche Interesse an der Wettbewerbspolitik unterscheidet sich aus zwei Gründen von der Wettbewerbsökonomik: zum einen liegt der akteursbezogene Fokus weniger auf den Unternehmen, sondern auf den politischen Akteuren und den Wettbewerbsbehörden. Zum anderen geht es nicht um die Analyse der Wirkung von Unternehmensstrategien an sich, sondern um Verfahren und institutionelle 
Merkmale der Wettbewerbspolitik. Die Entwicklung einer regelbasierten Wettbewerbspolitik ist dabei besonders interessant, zumal der grundsätzliche prozedurale und institutionelle Rahmen einer solchen in der Regel durch den Gesetzgeber definiert wird. Aber auch eine evidenzbasierte Wettbewerbspolitik ist von Interesse, da sich die Einzelentscheidungen auf eine regelbasierte Wettbewerbspolitik stützen und zur Entwicklung derselben beitragen. Der institutionelle Zuschnitt, der das Verhältnis von regel- und evidenzbasierter Wettbewerbspolitik bestimmt, und hierbei vor allem die Zuständigkeiten von Gesetzgeber, Regulierungsbehörden mit sektoriellem Zuschnitt und allgemeinen Wettbewerbsbehörden steht im Mittelpunkt der vergleichenden Analyse regulativer Regime (z.B. Coen \& Héritier 2005). Unter den Teilbereichen ist die Wettbewerbspolitik im Bereich staatliche Beihilfe für die politikwissenschaftliche Disziplin von originärem Interesse, da ihre Adressaten politische Akteure sind. So wurde beispielsweise die einschränkende Wirkung europäischer Beihilfekontrolle auf nationale wirtschafts- und industriepolitische Gestaltungsspielräume untersucht (Blauberger 2009). Aus politikökonomischer Sicht wird Wettbewerbspolitik als ein Prozess des Austarierens privater und öffentlicher Interessen verstanden (Wilks 2010). Infolgedessen favorisieren Politikwissenschaftler im Gegensatz zu Ökonominnen das Wohlfahrtkonzept der Konsumentenwohlfahrt, d.h. potentiell umverteilende Effekte werden als zentral erachtet. Abseits des disziplinären Mainstreams wurde das Politikfeld innerhalb eines kritischen Paradigmas der politischen Ökonomie vergleichsweise intensiv untersucht. Dies mag darin begründet liegen, dass sich die Wettbewerbspolitik normativ als „neoliberale”, marktschaffende Politik deuten lässt, die bestehende sozioökonomische Machtverhältnisse wenn nicht verstärkt, so doch zumindest erhält (Buch-Hansen \& Wigger 2011). Im Gegensatz zu einer solchen kritischen Perspektive stellen etablierte Analysekonzepte der politischen Ökonomie wie der regulative Staat oder der regulative Kapitalismus (Majone 1994, Levi-Faur \& Jordana 2005) die ideellen Grundlage der Wettbewerbspolitik nicht in Frage, sondern analysieren divergierende institutionelle und prozedurale Ausprägungen über Zeit, Sektoren und Länder hinweg. 


\section{Die politikwissenschaftliche Domäne: die Analyse von Institutionen und}

Prozessen der regulativen Politik

Eine systematische Wirkungsanalyse von regulierungs- und wettbewerbspolitischen Eingriffen steht nicht im Zentrum des politikwissenschaftlichen Interesses, sondern die Institutionen und Prozesse regulativer Politik. Der politikwissenschaftliche Beitrag in diesem Bereich wird im Folgenden vorgestellt, während der ökonomische Kommentar hierzu passende institutionenökonomische Überlegungen anstellt.

\subsection{Regulativer Staat, Regulative Governance, Regulativer Kapitalismus}

Seitdem Lowi in den 70er Jahren die regulative Politik als spezifische Politikarena analysiert hat, wurden in der politikwissenschaftliche Disziplin für deren institutionelle und prozedurale Ausprägungen diverse Begriffe geprägt und diskutiert. Wegweisend war dabei der Beitrag von Giandomenico Majone (1994). Majone hat Mitte der 1990er Jahre die These aufgestellt, dass in Nordamerika sowie in der Europäischen Gemeinschaft ein Übergang vom sogenannten „positiven“ zum „regulativen” Staat zu beobachten sei (Majone 1997). Während im positiven Staat im Rahmen des politischen Prozesses vor allem umverteilende Politik betrieben werde, zeichne sich der regulative Staat durch seine vornehmlich regelsetzende Rolle aus, wobei als regelsetzende Instanzen vermehrt sogenannte nichtmajoritäre Institutionen in Erscheinung treten würden, die außerhalb des durch Wahlen gekennzeichneten politischen Willensbildungsprozesses stünden. Diesen Trend sah Majone durch den zunehmenden Einfluss der Europäischen Gemeinschaft verstärkt, die aufgrund ihrer beschränkten finanziellen Mittel vor allem als regulativer Staat operiere. Im nationalen Kontext fußte diese Diagnose auf der Beobachtung, dass staatsnahe Sektoren privatisiert und liberalisiert wurden, und unabhängige Behörden mit der Aufgabe betraut wurden, diese neu geschaffenen Märkte zu steuern. Im Zuge der in der Politikwissenschaft zunehmend einflussreichen Governanceforschung (Mayntz 2005), die den Blick weitete für die empirisch beobachtbare Vielfalt regelsetzender Akteuren und Mechanismen, wurde der Begriff „regulative Governance” geprägt (Eckert 2011; Levi Faur 2007). Im Rahmen dieser Konzeptualisierung rückten insbesondere die Rolle privater Akteure, Mechanismen informeller und unverbindlicher Regelset- 
zung, sowie die Relevanz europäischer und internationaler Instanzen in den Blickpunkt. Diese umfassende Perspektive macht sich auch der Forschungsstrang zum „regulativer Kapitalismus" (Braithwaite 2008; Levi-Faur \& Jordana 2005) zu Eigen. Hinter dieser Begriffsbildung steht das Argument, dass im Zuge der expansiven Regulierungstätigkeit im nationalen und internationalen Kontext nicht von einem neoliberalen, durch Deregulierung gekennzeichneten, Wirtschaftsparadigma die Rede sein könne. Während die weiter reichenden Implikationen regulativer Politik für politische Ökonomien im Rahmen dieses Einführungskapitels nicht abgedeckt werden können, sollen zentrale theoretische und konzeptuelle Zugänge im Folgenden kurz vorgestellt werden. Um die institutionellen und prozeduralen Merkmale regulativer Politik zu analysieren, rekurrierte die politikwissenschaftliche Diskussion auf durch die Organisations- und Transaktionskostenökonomik inspirierte delegationstheoretische Zugänge. Im Unterschied zur ökonomischen Disziplin sind als Folge der Delegation zuvorderst Aspekte der Legitimität von Interesse, die insofern gesondert diskutiert werden sollen. Methode und Befund der politikwissenschaftlichen Forschung in diesem Bereich werden abschließend zusammengefasst.

\subsubsection{Delegationstheoretische Zugänge}

Um eine institutionelle Konstellation zu analysieren, in der regelsetzende Kompetenzen an nichtmajoritäre Institutionen abgegeben werden, hat sich die politikwissenschaftliche Diskussion in Europa primär auf Delegationstheorien gestützt und diese um neoinstitutionalistische theoretische Zugänge ergänzt (Thatcher \& Stone Sweet 2002). Einflussreich war hier vor allem der aus der Ökonomie importierte Analyserahmen einer Prinzipal-Agenten Beziehung (Moe 1990; Aghion \& Tirole 1997). Dieser Ansatz wurde zunächst in der US-amerikanischen Disziplin unter Rückgriff auf die Organisations- und Transaktionskostenökonomik entwickelt, und in der Folge in einer Vielzahl von rational choice basierten, quantitativen Studien angewandt (Pollack 2002). Die Grundannahmen des analytischen Zugriffs sind einfach und plausibel, und sind sicherlich mit ein Grund für die umfangreiche Rezeption des Ansatzes in der Disziplin: politische Akteure agieren als Prinzipal, indem sie ihre Autorität qua Amt dazu nutzen, um Befugnisse durch einen formalisierten Akt an nichtgewählte Institutionen, die Agenten, zu delegieren. Letztere verfügen inso- 
fern nur über Befugnisse, die ihnen durch diesen Akt der Delegation zugewiesen wurden. Funktionalistisch ist dieser Ansatz insofern, als dass angenommen wird, dass der Prinzipal ein Interesse an der Delegation hat, obwohl sie ihm Kosten im Kontext der Schaffung der institutionellen Arrangements sowie Folgekosten im Zuge der Überwachung und Kontrolle des Agenten abverlangt. Der Nutzen der Delegation - in der Form von Zeitersparnis, Expertise oder glaubhafter Versicherung muss also die Kosten der Delegation aufwiegen. Die unterschiedlichen Beweggründe der Delegation sowie hierauf abgestimmte institutionelle Arrangements wurden vielfach diskutiert. Aus einer Prinzipal-Agenten Perspektive ist dabei insbesondere von Interesse, wie viel Spielraum dem Agenten zugestanden wird. Klassischerweise liegt es im Interesse des politischen Prinzipals, den Wirkungsbereich des Agenten effektiv einzugrenzen und zu kontrollieren. Dies erfolgt zunächst durch die Festlegung eines klar umrissenen Zuständigkeitsbereiches, so dass die nicht-gewählten Akteure im Rahmen eines durch die politische Arena spezifizierten Mandates operieren. Dabei sollten im Idealfall die Präferenzen des Agenten mit denen des Prinzipals übereinstimmen, ein Abweichen wird als Delegationsverlust konzeptualisiert. Es mag aber auch im Interesse des Prinzipals liegen, dem Agenten weitreichende Gestaltungsspielräume zuzugestehen, um unabhängig von kurzfristigen politischen Motiven die Realisierung erwünschter Politikergebnisse sicher zu stellen. Zur Schaffung eines stabilen Regelungsumfeldes kann also eine Abkoppelung vom politischen Prozess erwünscht sein, da die Mechanismen des politischen Wettbewerbs zeitlich konsistenten Lösungen entgegenstehen (Dixit 1996: 65; Bendor et al. 2001: 260). Im Unterschied zu einer Agentenbeziehung im engeren Sinne hat Majone diese besondere Konstellation als Treuhänderbeziehung konzeptualisiert (Majone 2001: 103), und auf ihre empirische Relevanz etwa im Falle von Gerichten hingewiesen. Da eine empirische Abgrenzung dieser beiden Logiken schwer zu bewerkstelligen ist, macht es Sinn, diese nicht als Dichotomie, sondern vielmehr als Kontinuum zu verstehen (Eckert 2010). Grundlegendere Kritik am analytischen Rahmen einer Prinzipal-Agenten-Beziehung setzt dort an, wo eine rationalistische und funktionalistische Erklärung an ihre Grenzen stößt. Zwar ist es immer möglich, ex post institutionelle Arrangements als funktional zu erfassen, über die eigentlichen Beweggründe der beteiligten Akteure wird aber damit wenig ausgesagt. Hier setzen 
soziologisch- und historisch-institutionalistische Erklärungsansätze an, um etwa die Diffusion und Nachahmung von Delegationsmodellen zu durchleuchten, oder ihre Entwicklung im Zeitverlauf in den Blick zu nehmen (Thatcher \& Stone Sweet 2002).

\subsubsection{Input- und Outputlegitimität}

Neben der Frage, warum politische Akteure Entscheidungsbefugnisse im Bereich Wettbewerb und Regulierung an Institutionen außerhalb eines direkten politischen Zugriffs delegieren, widmet sich die politikwissenschaftliche Analyse auch den Folgen dieser Delegation. Sie tut dies aber aus einer legitimitätszentrierten Perspektive, während substantielle Politikergebnisse nur indirekt Berücksichtigung finden. Die viel rezipierte Unterscheidung zwischen Inputlegitimität, die sich bezieht auf die Herrschaft durch das Volk, und Outputlegitimität, die sich bezieht auf die Herrschaft für das Volk (Scharpf 1999: 16), trägt zu einer differenzierten Betrachtung der Problemstellung bei. Mit Bezug auf die Inputlegitimierung erscheint die Entpolitisierung durch Delegation zunächst problematisch, da sie die Kontrollmöglichkeiten durch gewählte, direkt legitimierte politische Akteure einschränkt. So könnten nicht-majoritäre Institutionen von politisch definierten, gemeinwohlorientierten Zielsetzungen abweichen und statt dessen von den Partikularinteressen der zu regulierenden Wirtschaftssektoren voreingenommen werden (regulatory capture; Stigler 1971). Um das Fehlen einer mittelbaren Legitimation durch die Rückbindung an die politische Arena auszugleichen, ist aus einer Inputperspektive die Sicherstellung einer prozeduralen Legitimation zentral. Mögliche Verfahren sind die Konsultation und Beteiligung relevanter Akteure, Regeln bezüglich der Transparenz in Entscheidungsprozessen, oder Berichterstattungspflichten. In diesem Kontext wurde betont, dass sich nichtmajoritäre Institutionen regelmäßig strengeren Standards unterwerfen, als dies im politischen Entscheidungsprozess der Fall sei (Thatcher \& Stone Sweet 2002: 19). Der Fokus der Rechtfertigung einer Abkoppelung vom politischen Prozess lag aber auf der Output-Legitimation (Scharpf 1999: 16-28). Diese liegt vor, wenn problemlösungsfähiges politisches Handeln gemeinwohlorientierte Ergebnisse zeitigt (Scharpf 1999: 16, 20). Ein ausreichendes Maß an Outputorientierter Legitimation hat das Potential, ein Fehlen an Input-orientierter Legitimation auszugleichen. Die Gründe, warum bestimmte Entscheidungsbereiche der 
direkten Kontrolle politischer Amtsinhaber entzogen werden, fußen auf delegationstheoretischen Überlegungen bezüglich wünschenswerter Politikergebnisse: zum Einen erscheint eine Delegation an Experten dort sinnvoll, wo es um komplexe Angelegenheiten geht, aber bezüglich der zu erzielenden Ergebnisse Konsens herrscht: hier fußt die Output-Legitimität vor allem auf einer erhöhten Effizienz durch die Reduktion von Entscheidungskosten im Vergleich zu den langwierigen Verfahren in der politischen Arena; zum Anderen sind Regulierungsbereiche wie die Geldpolitik oder investitionsintensive Sektoren identifiziert worden, in denen ein stabiles Regelumfeld und zeitlich konsistente Einzelentscheidungen besonders wichtig erscheinen, und insofern im Sinne einer glaubhaften Zusicherung von Politikergebnissen eine Entpolitisierung im Zuge einer Treuhänderbeziehung geboten ist. Aus politikwissenschaftlicher Sicht interessant ist die Diskussion um die Ergebnisse und Effekte regulativer Politik dort, wo ein erteiltes Mandat nicht eingehalten wird, und/oder das Resultat mit politischen Kosten verbunden ist. In diesem Kontext können Mechanismen der Rechenschaftspflicht greifen, und die politischen Akteure können versucht sein, die Verantwortung für unliebsame Ergebnisse regulativer Politik auf die ausführenden Behörden abzuwälzen. Ein solches Vorgehen birgt dann die Gefahr eines Bumerangeffektes, wenn die Letztverantwortung für den Output von der Wählerschaft in der politischen Arena gesehen wird (Hood 2002).

\subsubsection{Methoden und empirischer Befund}

Gemäß dem prioritären Interesse an institutionellen und prozeduralen Aspekten liegt auch der empirische Beitrag der (europäischen) politikwissenschaftlichen Forschung zur Regulierung vor allem in diesem Bereich: begründet durch eine delegationstheoretische Perspektive wurden die formale Unabhängigkeit sowie die Rechenschaftspflicht von Regulierungsbehörden in quantitativ vergleichenden Studien untersucht; die Diffusion von unabhängigen Regulierungsbehörden, sowie im Sektoren- und Ländervergleich ist ein weiterer Untersuchungsgegenstand; qualitativ ausgerichtete Studien haben sich den komplexen Akteurskonstellationen in einzelnen Politikbereichen und Ländern gewidmet, und institutionelle Veränderungen im Zeitverlauf untersucht. 
Wie Kompetenzen an nichtmajoritäre Institutionen delegiert werden, ist in der politikwissenschaftlichen Forschung intensiv untersucht worden. Einen zentralen Stellenwert nahm dabei das Konzept der formalen Unabhängigkeit ein, das als empirischer Annäherungswert an die Art des Delegationsverhältnisses verstanden werden kann: ein niedriges $M a ß$ an Unabhängigkeit entspricht eher der Agentenlogik, ein hohes Maß eher der Treuhänderlogik. Methodisch sind quantitativ vergleichende Untersuchungen zu unabhängigen Regulierungsbehörden in verschiedenen Ländern und Sektoren (Gilardi 2002) durch die frühere ökonomische Forschung zu unabhängigen Zentralbanken (Cukierman et al. 1992) inspiriert. Gilardi operationalisierte die Dimensionen formaler Unabhängigkeit auf der Basis von messbaren Einzelindikatoren, die er dann zu einem linearen Index auf einer Skala zwischen 0 (keine Unabhängigkeit) und 1 (maximale Unabhängigkeit) aggregierte. Diese methodische Vorgehensweise wurde vielfältig angewandt zur Untersuchung von unabhängigen Regulierungsbehörden auf nationaler und europäischer Ebene (Elgie \& McMenamin 2005; Gilardi 2005; Wonka \& Rittberger 2010). Ziel dieser vergleichenden Untersuchungen ist es, Muster der formalen Unabhängigkeit insbesondere im Länder- und Sektorenvergleich zu erfassen. In konzeptueller und methodischer Hinsicht ergaben sich in der Folge Kontroversen über eine angemessene Operationalisierung des Konzeptes der (formalen) Unabhängigkeit, und alternative Ansätze wurden in diesem Zusammenhang entwickelt (Hanretty \& Koop 2012). Aus politikwissenschaftlicher Sicht unbefriedigend erschien aber insbesondere der Fokus auf formalrechtlichen Kriterien, die die tatsächliche Praxis und informelle Prozesse berücksichtigen. Hier wurden Versuche angestellt, die de facto Unabhängigkeit entsprechend zu konzeptualisieren und zu operationalisieren, was beispielsweise in die Anwendung von QCA-Techniken mündete (Maggetti 2007). Aus einer soziologischinstitutionalistischen Perspektive wurde grundsätzlicher die Diffusion des institutionellen Modells unabhängiger Regulierung empirisch erfasst (Jordana et al. 2011), um der These des global diffundierenden regulativen Kapitalismus Nachdruck zu verleihen (Levi Faur 2005). Neben diesen quantitativ orientierten Studien haben sich zahlreiche politikwissenschaftliche Publikationen der qualitativen Analyse einiger weniger Fälle gewidmet. Hierbei wurden neben dem Verhältnis zwischen der politischen Arena und den unabhängigen Behörden (Thatcher 2005; Eckert 2010) auch 
die Beziehungen zwischen den Regulierern und den regulierten Marktteilnehmern detailliert untersucht (Coen 2005), oder zwischen diesen beiden Polen als, intermediäre' Institutionen konzeptualisiert (Braun 1993). Der Beitrag dieser Vergleichsund Einzelfallstudien lag beispielsweise darin, aus einer historischinstitutionalistischen Perspektive Veränderungen im Zeitverlauf nachzuvollziehen oder komplexe Konstellationen im europäischen Mehrebenensystem zu erfassen (z.B. Thatcher \& Coen 2008).

\section{2. Ökonomischer Kommentar}

Bereits in der Darstellung der politikwissenschaftlichen Sicht im vorigen Kapitel wurde deutlich, dass diese auch auf ökonomischen Grundlagen beruht, insbesondere auf den Strömungen der sog. Neuen Politischen Ökonomie (bzw. Public Choice) und der Institutionenökonomik. Auch wenn diese nicht im Zentrum des industrieökonomisch geprägten Denkens der (Mainstream) Wettbewerbs- und Regulierungsökonomik stehen, so gibt es freilich ökonomische Literatur, welche sich mit solchen Fragestellungen befasst. Diese wird oftmals als komplementär zur Wirkungsanalyse betrachtet (Budzinski 2008a, 2013c). Eine deutliche Übereinstimmung zwischen der wirtschafts- und politikwissenschaftlichen Sicht gibt es dabei in einem ersten Feld, nämlich der Frage der Unabhängigkeit von Wettbewerbs- und Regulierungsbehörden, auch wenn - der Diskussion um Geldwertstabilität und zentrale Notenbanken folgend - hier in der Ökonomik eher die Outputlegitimität im Fokus steht (Duijm 1999; Budzinski 2013b). Der Gedankenwelt der Public ChoiceForschung folgend wurde zweitens insbesondere in der US-amerikanischen Literatur die Frage nach den Eigeninteressen von Regulierern sowie möglichen Problemen eines regulatory capture gestellt (für eine neuere Zusammenfassung der eher radikalen Positionen siehe McChesney et al. 2015), woraus sich einerseits eine starke und durchaus auch radikale Deregulierungsströmung speiste (basierend auf der theoretischen und empirischen Erkenntnis, dass selbst dort, wo theoretisch durch Regulierung Wohlfahrtsvorteile erzielt werden können, reale Regulierung durch reale Regulierer oftmals eher zu Verschlechterungen führen; Haucap et al. 2013), andererseits aber auch Regulierungsinnovationen wie die Konzepte der Anreizregulierung oder der Sunsetregulierungen hervorgingen. 
Ein drittes institutionelles Thema, das auch von ökonomischer Seite analysiert wird, ist die Kompetenzabgrenzung zwischen Behörden, beispielsweise zwischen Regulierungs- und Wettbewerbsbehörden (bspw. Haucap \& Uhde 2008) oder auch im Hinblick auf Zentralität und Dezentralität von Regulierungen (bspw. Heine \& Kerber 2007). In Verbindung mit dem aus der Politikwissenschaft importierten Thema der Mehrebenengovernancesysteme spielen Fragen der Kompetenzabgrenzung insbesondere auch bei der Diskussion um internationale Wettbewerbspolitikprobleme und -institutionen eine wesentliche Rolle (bspw. Budzinski 2015). Schließlich sei viertens noch auf einen Themenkomplex verwiesen, der sowohl rechts- und wirtschafts- als auch politikwissenschaftliche Aspekte enthält: bei der Frage, wie wettbewerbspolitische Regeln und Verfahren ausgestaltet werden sollten, ist in jüngerer Zeit eine ökonomische Literatur entstanden, welche die alte juristische Dichotomie zwischen Per se-Regeln und Rule-of-Reason-Standards um neue inhaltliche wie auch modelltheoretische Facetten bereichert (bspw. Christiansen \& Kerber 2006; Kerber et al. 2008; Budzinski 2010; Brennan 2014). Dahinter steht letztendlich die auch politikwissenschaftlich relevante Frage nach dem Trade-off zwischen allgemeinen Regeln und Einzelfallbesonderheiten. Innerhalb der Ökonomik ist hiermit die sog. Law \& Economics-Strömung angesprochen (Budzinski und Haucap 2015). Hinsichtlich der Methoden gewinnt in der Ökonomik auch in diesen Bereichen die empirische, ökonometrische Wirtschaftsforschung an Boden (bspw. Voigt 2011, 2013).

\section{Schlussfolgerungen}

Die eingangs erwartete Komplementarität einer ökonomischen und politikwissenschaftlichen Sichtweise auf Wettbewerb und Regulierung hat sich im Laufe unseres interdisziplinären Beitrages bestätigt und substantiell verfestigt. Die Wettbewerbsökonomik ermöglicht in ihrer modernen Hauptströmung eine ausdifferenzierte Wirkungsanalyse von Unternehmensstrategien in den diversen Teilbereichen der Wettbewerbspolitik, also der Kartellpolitik, der Fusionskontrolle und der Missbrauchsaufsicht über marktbeherrschende Unternehmen. In ähnlicher Weise fokussiert die Regulierungsökonomik darauf, Regulierungsnotwendigkeit zu identifizieren (Theorie des Marktversagens) und zu untersuchen, welche Regulierungsinstrumente zu effizienten, wohlfahrtsteigernden Ergebnissen führen. In beiden Berei- 
chen ist dabei eine umfangreiche Literatur entstanden, welche die große Vielfalt von Wettbewerbs- und Regulierungsproblemen reflektiert und teilweise bis auf die Ebene der Modellierung der Besonderheiten einzelner Fälle ins Detail geht. Daher war es uns auch in diesem Beitrag nicht möglich, diese Literatur in all ihren Facetten darzustellen und aufzuführen, sondern wir mussten uns überwiegend auf den Verweis auf zusammenfassende Übersichtsbeiträge beschränken. Gemäß der Zentralität des Gegenstandes ist die Wettbewerbs- und Regulierungsökonomik aus interdisziplinärer Perspektive äußerst kleinteilig ausgerichtet und methodisch ausgereift.

Hingegen sind politikwissenschaftliche Reflektionen zur Wettbewerbspolitik ein eher unterentwickeltes Feld, das aus Sicht diverser Subdisziplinen einen zentraleren Stellenwert in der Forschung verdient: in der vergleichenden Politikwissenschaft können die institutionellen Ausprägungen im Länder- und Sektorenvergleich aus qualitativer wie auch quantitativer Sicht stärker Berücksichtigung finden - hier haben unabhängige Regulierungsbehörden und Regulierungsregime im Fokus der Analyse gestanden. In der vergleichenden, sowie internationalen politischen Ökonomie sollten die Rückwirkungen einer expandierenden und diffundierenden Wettbewerbspolitik auf nationale Ökonomien sowie auf das internationale ökonomische System vermehrt in den Blick genommen werden, auch jenseits einer dezidiert kritischen Analyse. Generell bietet sich ein Fokus auf die politische Steuerung von Unternehmensstrategien im Rahmen der Wettbewerbspolitik an, die bis dato zu sehr als Variante der regulativen Politik mitbehandelt wurde und als eigenständiges Untersuchungsfeld weitgehend den Wirtschafts- und Rechtswissenschaften überlassen wurde. Hingegen haben Institutionen und Prozesse dessen, was durch die Regulierungsökonomik abgedeckt wird, viel stärker im Fokus der politikwissenschaftlichen Untersuchung gestanden. Dies zeigt sich schon darin, dass das Adjektiv ,regulativ' zur Charakterisierung zentraler Konzepte wie Policy, Staat, Kapitalismus und Governance vielfach Verwendung fand. Politikwissenschaftlerinnen haben die Delegation von Regelungskompetenzen an nichtgewählte Institutionen aus delegationstheoretischer und institutionalistischer Perspektive intensiv untersucht, und deren Konsequenzen für die Legitimität politischer Prozesse erörtert. Der empirisch-analytische 
Zugriff war dabei weitaus mehr auf den Vergleich ausgerichtet als in der Ökonomik, wobei als Vergleichseinheiten Länder, Politikbereiche, oder auch Politikebenen untersucht wurden. Von besonderem Interesse ist dabei die Verlagerung von regulierungs- wettbewerbspolitischen Kompetenzen auf die europäische oder internationale Ebene im Mehrebenenkontext. Letzterer findet auch zunehmend in der Ökonomik Beachtung. Weitere Schnittmengen mit der Ökonomie ergeben sich vor allem im Bereich der Neuen Politischen Ökonomie und der Institutionenökonomik. Hier werden ähnliche Themen wie in der Politikwissenschaft thematisiert (Unabhängigkeit von Regulierungs- und Wettbewerbsbehörden, Politökonomie der Regulierer, institutionelle Schnittstellen in Mehr-Ebenen-Regulierungs- oder Wettbewerbspolitiksystemen, etc.), allerdings mit ökonomischen Methoden und mit Blick auf Wohlfahrtsziele und Effizienzwirkungen.

Schließlich bleibt abschließend festzuhalten, dass sich ein interdisziplinärer Blick auf die Problematik Wettbewerb und Regulierung nicht auf die wirtschafts- und politikwissenschaftlichen Perspektiven beschränken kann und sollte. Hinzukommen muss die rechtswissenschaftliche Perspektive, da sowohl Wettbewerbspolitik als auch Regulierung stets die Anwendung und Durchsetzung von Rechtsnormen implizieren und ein erheblicher Teil des realwissenschaftlichen Phänomens Wettbewerb und Regulierung durch juristische Erwägungen und Aspekte bestimmt wird. Dies gilt gerade auch für normative Aussagen der Ökonomik und der Politikwissenschaft, welche nicht selten an juristische Grenzen stoßen. Umgekehrt bedarf natürlich auch die rechtswissenschaftliche Sicht der Kenntnis wirtschaftswissenschaftlicher Wirkungen und politikwissenschaftlicher Erkenntnisse, um den Bezug zu den durch Wettbewerbspolitik und Regulierung zu lösenden Problemen nicht zu verlieren. 


\section{Literaturverzeichnis}

Aghion, Paul and Jean Tirole. 1997. Formal and Real Authority in Organizations. Journal of Political Economy 105: 1-29.

Aydin, Umut and Kenneth P. Thomas. 2012. The Challenges and Trajectories of EU Competition Policy in the Twenty-first Century. Journal of European Integration 34(6): 531-47.

Bendor, Jonathan, Amihai Glazer and Thomas Hammond. 2001. Theories of Delegation. Annual Review of Political Science 4: 235-269.

Bishop, Simon and Mike Walker. 2010. The Economcis of EC Competition Law: Concepts, Application and Measurement. $3^{\text {rd }}$ edition. London: Sweet \& Maxwell.

Blair, Roger D. and D. Daniel Sokol. Hrsg. 2015. The Oxford Handbook of International Antitrust Economics. Vol. 1 und 2. Oxford: Oxford University Press.

Blauberger, Michael. 2009. Of 'Good' and 'Bad' Subsidies: European State Aid Control through Soft and Hard Law. West European Politics 32(4): 719-737.

Brennan, Timothy F. 2014. Is Complexity in Antitrust a Virtue? The AccuracySimplicity Tradeoff. The Antitrust Bulletin 59 (4): 827-853.

Buch-Hansen, Hubert and Angela Wigger. 2011. The Politics of European Competition Regulation. A Critical Political Economy Perspective. London, New York: Routledge.

Braithwaite, John. 2008. Regulatory Capitalism. How it Works, Ideas for Making it Work Better. Cheltenham: Edward Elgar.

Braun, Dietmar. 1993. Who Governs Intermediary Agencies? Principal-Agent Relations in Research Policy-Making. Journal of Public Policy 13(2): 135-162.

Buccirossi, Paolo. Hrsg. 2008. Handbook of Antitrust Economics. Cambridge, Mass.: The MIT Press.

Budzinski, Oliver. 2008a. Monoculture versus Diversity in Competition Economics. Cambridge Journal of Economics 32 (2): 295-324.

Budzinski, Oliver. 2008b. Wettbewerbsfreiheit" und "More Economic Approach": Wohin steuert die Europäische Wettbewerbspolitik? In Quo vadis Wirtschaftspolitik?, Hrsg. Marina Grusevaja, Christoph Wonke, Ulrike Hösel und Malcolm H. Dunn, S. 15-38. Frankfurt a.M.: Lang.

Budzinski, Oliver. 2010. An Institutional Analysis of the Enforcement Problems in Merger Control. European Competition Journal 6 (2): 445-474.

Budzinski, Oliver. 2011. Modern Industrial Economics and Competition Policy. In Competition Policy and the Economic Approach: Foundations and Limitations. Hrsg. Josef Drexl, Wolfgang Kerber und Rupprecht Podszun. pp. 111-138. Cheltenham: Elgar.

Budzinski, Oliver. 2012. Empirische Ex-Post Evaluation wettbewerbspolitischer Entscheidungen: Methodische Anmerkungen. In Methodische Grundlagen der empirischen Institutionenökonomik, Hrsg. Theresia Theurl, S. 45-71. Berlin: Duncker \& Humblot. 
Budzinski, Oliver. 2013a. Impact Evaluation of Merger Control Decisions. European Competition Journal 9 (1): 199-224.

Budzinski, Oliver. 2013b. Würde eine unabhängige Wettbewerbsbehörde eine bessere Wettbewerbspolitik machen? In Unabhängige staatliche Institutionen in der Demokratie, Hrsg. Theresia Theurl, S. 157-187. Berlin: Duncker \& Humblot.

Budzinski, Oliver. 2013c. Zur Bedeutung der Ordnungsökonomik für eine moderne, ökonomiebasierte Wettbewerbspolitik. In Das weite Feld der Ökonomik, Festschrift zu Ehren von Prof. Dr. Karl-Hans Hartwig, Hrsg. Ingo Pies, S. 145-167. Stuttgart: Lucius \& Lucius.

Budzinski, Oliver. 2015. International Antitrust Institutions. In The Oxford Handbook of International Antitrust Economics. Vol. 1, Hrsg. Roger D. Blair and D. Daniel Sokol, pp. 119-146. Oxford: Oxford University Press.

Budzinski, Oliver und Justus Haucap. Hrsg. 2015. Recht und Ökonomie. BadenBaden: Nomos. Im Druck.

Budzinski, Oliver and Isabel Ruhmer. 2010. Merger Simulation in Competition Policy. Journal of Competition Law \& Economics 6 (2): 277-320.

Choi, Jay Pill and Heiko Gerlach. 2015. Cartels and Collusion: Economic Theory and Experimental Economics. In The Oxford Handbook of International Antitrust Economics. Vol. 2, Hrsg. Roger D. Blair and D. Daniel Sokol, pp. 415-441. Oxford: Oxford University Press.

Christiansen, Arndt. 2010. Der "More Economic Approach" in der EUFusionskontrolle: Entwicklungen, konzeptionelle Grundlagen und kritische Analyse. Frankfurt a.M.: Lang.

Christiansen, Arndt and Wolfgang Kerber. 2006. Competition Policy with Optimally Differentiated Rules Instead of "Per se Rules vs. Rule of Reason". Journal of Competition Law and Economics 2 (2): 215-244.

Cini, Michele and Lee McGowan. 2008. Competition Policy in the European Union. 2nd edition. Basingstoke: Palgrave Macmillan.

Clark, John M. 1961. Competition as a Dynamic Process. Washington D.C.: Brookings Institution.

Coen, David. 2005. Business-Regulatory Relations: Learning to Play Regulatory Games in European Utility Markets. Governance: An International Journal of Policy, Administration and Institutions 18(3): 375-398.

Coen, David and Adrienne Héritier. Hrsg. 2005. Refining Regulatory Regimes. Utilities in Europe. Cheltenham: Edward Elgar.

Cukierman, Alex, Steven B. Webb and Bilin Neyapti. 1992. Measuring the independence of central banks and its effects on policy outcomes. World Bank Economic Review 6(3): 353-398.

Czada, Roland und Susanne Lütz. 2003. Einleitung - Probleme, Institutionen und Relevanz regulativer Politik. In Regulative Politik, Hrsg. Roland Czada, Susanne Lütz und Stefan Mette, S. 13-34. Opladen: Leske und Budrich. 
Dixit, Avinash K. 1996. The Making of Economic Policy: a transaction-cost politics perspective. Cambridge, Mass.: The MIT Press.

Doern, G. Bruce and Stephen Wilks. 1996. Comparative Competition Policy: National Institutions in a Global Market. Oxford: Oxford University Press.

Doose, Anna Maria. 2014. Methods for Calculating Cartel Damages. Zeitschrift für Wettbewerbsrecht 12 (3): 282-299.

Duijm, Bernhard. 1999. Die Unabhängigkeit von Kartellbehörden. ORDO 50: 323344.

Eckert, Sandra. 2010. Between Commitment and Control: Varieties of Delegation in the European Postal Sector. Journal of European Public Policy 17(8): 1231-1252.

Eckert, Sandra. 2011. European Regulatory Governance. In Handbook on the Politics of Regulation, Hrsg. David Levi-Faur, pp. 513-524. Cheltenham: Edward Elgar Publishing.

Elgie, Robert and Ian McMenamin. 2005. Credible Commitment, Political Uncertainty or Policy Complexity? Explaining Variations in the Independence of Nonmajoritarian Institutions in France. British Journal of Political Science 35(3): 531 548.

Elzinga, Kenneth G. and David E. Mills. 2015. Predatory Pricing. In The Oxford Handbook of International Antitrust Economics. Vol. 2, Hrsg. Roger D. Blair and D. Daniel Sokol, pp. 40-61. Oxford: Oxford University Press.

Eucken, Walter. 1952. Grundsätze der Wirtschaftspolitik. Tübingen: Mohr Siebeck.

Ewald, Christian. 2011. Ökonomie im Kartellrecht: Vom more economic approach zu sachgerechten Standards forensischer Ökonomie. Zeitschrift für Wettbewerbsrecht 9 (1): 15-47.

Franck, Jens-Uwe. 2015. Wettbewerbsschutz durch Kartellrecht: Normative Grenzen einer an ökonomischen Rationalitäten ausgerichteten Marktordnung. In Recht und Ökonomie, Hrsg. Oliver Budzinski und Justus Haucap, in Vorbereitung. Baden-Baden: Nomos.

Fritsch, Michael. 2014. Marktversagen und Wirtschaftspolitik. 9. Auflage. München: Vahlen.

Gilardi, Fabrizio. 2002. Policy credibility and delegation to independent regulatory agencies: a comparative empirical analysis. Journal of European Public Policy 9(6): 873-893.

Gilardi, Fabrizio. 2005. The Formal Independence of Regulators: A Comparison of 17 Countries and 7 Sectors. Swiss Political Science Review 11(1): 139-167.

Hanretty, Chris and Christel Koop. 2012. Measuring the formal independence of regulatory agencies. Journal of European Public Policy 19 (2): 198-216.

Haucap, Justus und Ingo Schmidt. 2013. Wettbewerbspolitik und Kartellrecht: eine interdisziplinäre Einführung. 10. Auflage. München: Oldenbourg. 
Haucap, Justus und André Uhde. 2008. Regulierung und Wettbewerbsrecht in liberalisierten Netzindustrien aus institutionenökonomischer Perspektive. ORDO 59: 237-262.

Haucap, Justus, Mirjam R. Lange und Christian Wey. 2013. Nemo Omnibus Placet: exzessive Regulierung und staatliche Willkür. In Akzeptanzprobleme der Marktwirtschaft: Ursachen und wirtschaftspolitische Konsequenzen, Hrsg. Theresia Theurl, S. 145-167. Berlin: Duncker \& Humblot.

Hayek, Friedrich A. von. 1945. The Use of Knowledge in Society. American Economic Review 35 (4): 519-530.

Hayek, Friedrich A. von. 1968. Der Wettbewerb als Entdeckungsverfahren. Kieler Vorträge Neue Folge 56. Kiel: Institut für Weltwirtschaft.

Hellwig, Martin. 2006. Effizienz oder Wettbewerbsfreiheit? Zur normativen Grundlegung der Wettbewerbspolitik. In Recht und spontane Ordnung, Hrsg. Christoph Engel und Wernhard Möschel, S. 231-268, Baden-Baden: Nomos.

Heine, Klaus und Wolfgang Kerber. Hrsg. 2007. Zentralität und Dezentralität von Regulierung in Europa. Stuttgart: Lucius \& Lucius.

Heinelt, H. 2003. Politikfelder: Machen Besonderheiten von Policies einen Unterschied? In Lehrbuch der Politikfeldanalyse, Hrsg. Schubert, Klaus und Nils Bandelow, S. 239-256, München: Oldenbourg.

Héritier, Adrienne. 1987. Policy Analyse - Eine Einführung. Frankfurt/ New York: Campus Verlag.

Hood, Christopher. 2002. The Risk Game and the Blame Game. Government and Opposition 37(1): 15-37.

Inderst, Roman, Frank Maier-Rigaud and Ulrich Schwalbe. 2014. Umbrella Effects. Journal of Competition Law and Economics 10 (3): 739-763.

Jayaratne, Jith und Janusz Ordover. 2015. Coordinated Effects: Evolution of Practice and Theory. In The Oxford Handbook of International Antitrust Economics. Vol. 1, Hrsg. Roger D. Blair and D. Daniel Sokol, pp. 509-528. Oxford: Oxford University Press.

Jordana, Jacint, David Levi-Faur and Xavier Fernández i Marín. 2011. The Global Diffusion of Regulatory Agencies: Channels of Transfer and Stages of Diffusion. Comparative Political Studies 44(10): 1343-69.

Keating, Bryan and Robert D. Willig. 2015. Unilateral Effects. In The Oxford Handbook of International Antitrust Economics. Vol. 1, Hrsg. Roger D. Blair and D. Daniel Sokol, pp. 466-508. Oxford: Oxford University Press.

Kerber, Wolfgang. 2007. Wettbewerbspolitik. In Vahlens Kompendium der Wirtschaftstheorie und Wirtschaftspolitik. Band 2, S. 369-434, München: Vahlen.

Kerber, Wolfgang und Ulrich Schwalbe. 2007. Ökonomische Grundlagen des Wettbewerbsrechts. In Münchener Kommentar zum Europäischen und Deutschen Wettbewerbsrecht. Bd. I, Hrsg. Franz Jürgen Säcker und Frank Montag, S. 238430. München: C.H. Beck. 
Kerber, Wolfgang, Jürgen-Peter Kretschmer and Georg von Wangenheim. 2008. Optimal Sequential Investigation Rules in Competition Law. Working Paper. Marburg: Universität Marburg.

Levenstein, Margaret C. and Valerie Y. Suslow. 2015. Cartels and Collusion: Empirical Evidence. In The Oxford Handbook of International Antitrust Economics. Vol. 2, Hrsg. Roger D. Blair and D. Daniel Sokol, pp. 442-463. Oxford: Oxford University Press.

Levi-Faur, David. 2005. The Global Diffusion of Regulatory Capitalism. Annals of the American Academy of Political and Social Science 598: 12-32.

Levi-Faur, David. 2007. Regulatory Governance. In Europeanization. New Research Agendas, Hrsg. Paolo Graziano und Maarten P. Vink, pp. 102-114. Basingstoke: Palgrave Macmillan.

Levi-Faur, David and Jacint Jordana. 2005. Globalizing Regulatory Capitalism. The ANNALS of the American Academy of Political and Social Science 598(1): 6-9.

Lowi, Theodore J. 1972. Four Systems of Policy, Politics, and Choice. Public Administration Review 32(4): 298-310.

Maggetti, Martino. 2007. De facto Independence after Delegation: a fuzzy-set analysis. Regulation \& Governance 1: 271-294.

Majone, Giandomenico. 1994. The Rise of the Regulatory State in Europe. West European Politics 17(3): 77-101.

Majone, Giandomenico. 1997. From the Positive to the Regulatory State: Causes and Consequences of Changes in the Mode of Governance. Journal of Public Policy 17(2): 139-167.

Majone, Giandomenico. 2001. Two Logics of Delegation: Agency and Fiduciary Relations in EU Governance. European Union Politics 2(1): 103-122.

Mayntz, Renate. 2005. Governance Theory als Fortentwicklung der Steuerungstheorie? In Governance-Forschung: Vergewisserung über Stand und Entwicklungslinien, Hrsg. Gunnart F. Schuppert, S. 11-20. Nomos: Baden-Baden.

McChesney, Fred S., Michael Reksulak und William F. Shughart. 2015. Competition Policy in Public Choice Perspective. In The Oxford Handbook of International Antitrust Economics. Vol. 1, Hrsg. Roger D. Blair and D. Daniel Sokol, pp. 147-171. Oxford: Oxford University Press.

Moe, Terry M. 1990. Political Institutions. The Neglected Side of the Story. Journal of Law, Economics and Organization 6(Special Issue): 213-253.

Motta, Massimo. 2004. Competition Policy: Theory and Practice. Cambridge: Cambridge University Press.

Neven, Damien. 2006. Competition Economics and Antitrust in Europe. Economic Policy 21 (48): 741-791.

Orbach, Barak und Raphael Avraham. 2015. Squeezing Claims: Refusal to Deal, Essential Facilities, and Price Squeezes. In The Oxford Handbook of International Antitrust Economics. Vol. 2, Hrsg. Roger D. Blair and D. Daniel Sokol, pp. 120131. Oxford: Oxford University Press. 
Pollack, Mark A. 2002. Learning from the Americanists (Again): Theory and Method in the Study of Delegation. West European Politics 25 (1): 200-219.

Röller, Lars-Hendrik. 2005. Economic Analysis and Competition Policy Enforcement in Europe. In Modelling European Mergers, Hrsg. Peter A.G. van Bergeijk and Erik Kloosterhuis, pp. 13-26, Cheltenham: Elgar.

Röpke, Wilhelm. 1949. Civitas humana - Grundfragen der Gesellschafts- und Wirtschaftsreform. 3. Auflage. Erlenbach-Zürich.

Rubinfeld, Daniel L. 2010. Econometric Issues in Antitrust Analysis. Journal of Institutional and Theoretical Economics 166 (1): 62-77.

Salinger, Michael A. 2015. Vertical Mergers. In The Oxford Handbook of International Antitrust Economics. Vol. 1, Hrsg. Roger D. Blair and D. Daniel Sokol, pp. 551-585. Oxford: Oxford University Press.

Scharpf, Fritz W. 1999. Regieren in Europa: Effektiv und demokratisch? Frankfurt/Main, New York: Campus.

Scheffman, David T. and Richard S. Higgins. 2015. Raising Rivals' Costs. In The Oxford Handbook of International Antitrust Economics. Vol. 2, Hrsg. Roger D. Blair and D. Daniel Sokol, pp. 62-71. Oxford: Oxford University Press.

Schmidt, Ingo und André Schmidt. 2006. Europäische Wettbewerbspolitik und Beihilfenkontrolle. 2. Auflage. München: Vahlen.

Schwalbe, Ulrich und Daniel Zimmer. 2011. Kartellrecht und Ökonomie: Moderne ökonomische Ansätze in der europäischen und deutschen Zusammenschlusskontrolle. 2. Auflage. München: Beck.

Stigler, George J. 1971. The Theory of Economic Regulation. The Bell Journal of Economics and Management Science 2(1): 3-21.

Thatcher, Mark and Alec Stone Sweet. 2002. Theory and Practice of Delegation to Non-Majoritarian Institutions. West European Politics 25(1): 1-22.

Thatcher, Mark. 2005. The Third Force? Independent Regulatory Agencies and Elected Politicians in Europe. Governance. An International Journal of Policy, Administration and Institutions 18(3): 347-373.

Thatcher, Mark and David Coen. 2008. Reshaping European regulatory space: an evolutionary analysis. West European Politics 31(4): 806-836.

Tirole, Jean. 2005. The Analysis of Tying Cases: A Primer. Competition Policy International 1 (1): 1-25.

Töller, Annette. 2008. Wettbewerbspolitik. In Politikfelder im EUMehrebenensystem, Hrsg. H. Heinelt und M. Knodt, S. 115-139, Baden-Baden: Nomos.

Van den Bergh, Roger J. and Peter D. Camesasca. 2006. European Competition Law and Economics: A Comparative Perspective. $2^{\text {nd }}$ edition. London: Thomson Sweet \& Maxwell. 
Vanberg, Viktor J. 1999. Markets and Regulation: On the Contrast Between FreeMarket Liberalism and Constitutional Liberalism. Constitutional Political Economy 10 (3): 219-243.

Vanberg, Viktor J. 2000. Economic Constitutions, Protectionism, and Competition among Jurisdictions. In Competition and Structure - The Political Economy of Collective Decisions, Hrsg. G. Galeotti, Pierre Salmon and R. Wintrobe, pp. 364385, Cambridge: Cambridge University Press.

Vanberg, Viktor J. 2011. Consumer Welfare, Total Welfare and Economic Freedom: On the Normative Foundations of Competition Policy. In Competition Policy and the Economic Approach, Hrsg. Josef Drexl, Wolfgang Kerber and Rupprecht Podszun, pp. 44-71. Cheltenham: Elgar.

Voigt, Stefan. 2011. Empirical Constitutional Economics: Onward and Upward? Journal of Economic Behavior and Organization 80 (2): 319-330.

Voigt, Stefan. 2013. How (Not) To Measure Institutions. Journal of Institutional Economics 9 (1): 1-26.

Weizsäcker, C. Christian von. 2007. Konsumentenwohlfahrt und Wettbewerbsfreiheit: Über den tieferen Sinn des „Economic Approach". Wirtschaft und Wettbewerb 57 (11): 1078-1084.

White, Lawrence J. 2015. Monopoly and Dominant Firms: Antitrust Economics and Policy Approaches, In The Oxford Handbook of International Antitrust Economics. Vol. 1, Hrsg. Roger D. Blair and D. Daniel Sokol, pp. 313-344. Oxford: Oxford University Press.

Wilks, Stephen. 2010. Competition Policy. In The Oxford Handbook of Business and Government, Hrsg. D. Coen et al., pp. 730-756. Oxford: Oxford University Press.

Wonka, Arndt and Berthold Rittberger. 2010. Paths to independence: Determinants of institutional independence of twenty-nine EU agencies. West European Politics 33(4): 730-752. 


\section{Diskussionspapiere aus dem Institut für Volkswirtschaftslehre der Technischen Universität IImenau}

Nr. 42 Steinrücken, Torsten; Jaenichen, Sebastian: Wer ist wirklich reich? - Zu Problemen der Wohlfahrtsmessung durch das Bruttoinlandsprodukt, April 2005.

Nr. 43 Steinrücken, Torsten; Jaenichen, Sebastian: Wo bleiben die Subventionssteuern? - Probleme des Beihilfenrechts und ein alternatives Regulierungskonzept, Mai 2005.

Nr. 44 Jaenichen, Sebastian; Steinrücken, Torsten; Schneider, Lutz: Zu den ökonomischen Wirkungen gesetzlicher Feiertage - Eine Diskussion unter besonderer Berücksichtigung der Arbeitszeitpolitik, Juni 2005.

Nr. 45 Kuchinke, Björn A.: Qualitätswettbewerb zwischen deutschen Akutkrankenhäusern unter besonderer Berücksichtigung von DRG und Budgets, Juni 2005.

Nr. 46 Kuchinke, Björn A.; Walterscheid, Heike: Wo steht der Osten? Eine ökonomische Analyse anhand von Wohlfahrts- und Happinessindikatoren, Juni 2005.

Nr. 47 Kuchinke, Björn A.; Schubert, Jens M.: Staatliche Zahlungen an Krankenhäuser: Eine juristische und ökonomische Einschätzung nach Altmark Trans und der Entscheidung der Kommission vom 13.7.2005, August 2005.

Nr. 48 Steinrücken, Torsten; Jaenichen, Sebastian: Überkapazitäten zur Absicherung politischer Risiken und Instrumente finanzwirtschaftlicher Gegensteuerung, November 2005.

Nr. 49 Jaenichen, Sebastian; Steinrücken, Torsten: Opel, Thüringen und das Kaspische Meer, Januar 2006.

Nr. 50 Kallfaß, Hermann H.: Räumlicher Wettbewerb zwischen Allgemeinen Krankenhäusern, Februar 2006.

Nr. 51 Sickmann, Jörn: Airport Slot Allocation, März 2006.

Nr. 52 Kallfaß, Hermann H.; Kuchinke, Björn A.: Die räumliche Marktabgrenzung bei Zusammenschlüssen von Krankenhäusern in den USA und in Deutschland: Eine wettbewerbsökonomische Analyse, April 2006.

Nr. 53 Bamberger, Eva; Bielig, Andreas: Mehr Beschäftigung mittels weniger Kündigungsschutz? Ökonomische Analyse der Vereinbarungen des Koalitionsvertrages vom 11. 11. 2005, Juni 2006. 
Nr. 54 Jaenichen, Sebastian; Steinrücken, Torsten: Zur Ökonomik von Steuergeschenken - Der Zeitverlauf als Erklärungsansatz für die effektive steuerliche Belastung, Dezember 2006.

Nr. 55 Jaenichen, Sebastian; Steinrücken, Torsten: Wirkt eine Preisregulierung nur auf den Preis? Anmerkungen zu den Wirkungen einer Preisregulierung auf das Werbevolumen, Mai 2007.

Nr. 56 Kuchinke, B. A.; Sauerland, D.; Wübker, A.: Determinanten der Wartezeit auf einen Behandlungstermin in deutschen Krankenhäusern - Ergebnisse einer Auswertung neuer Daten, Februar 2008.

Nr. 57 Wegehenkel, Lothar; Walterscheid, Heike: Rechtsstruktur und Evolution von Wirtschaftssystemen - Pfadabhängigkeit in Richtung Zentralisierung?, Februar 2008.

Nr. 58 Steinrücken, Torsten; Jaenichen, Sebastian: Regulierung und Wohlfahrt in einem Modell mit zwei Aktionsparametern, März 2008.

Nr. 59 Lehnert, Ninja M.: Externe Kosten des Luftverkehrs - Ein Überblick über den aktuellen Stand der Diskussion, April 2008.

Nr. 60 Walterscheid, Heike: Reformbedarf etablierter Demokratien im Kontext dezentralisierter Gesellschaftssysteme - Grundlegende Hindernisse bei Steuersystemreformen", April 2010.

Nr. 61 Walterscheid, Heike; Wegehenkel, Lothar: Kostenstruktur, Zahlungsbereitschaft und das Angebot von Mediengütern auf Medienmärkten, Juni 2008.

Nr. 62 Walterscheid, Heike; Wegehenkel, Lothar: Wohlstand der Nationen und handlungsrechtliche Struktur eines Gesellschaftssystems, September 2008.

Nr. 63 Dewenter, Ralf; Haucap, Justus; Wenzel, Tobias: Indirect Network Effects with Two Salop Circles: The Example oft the Music Industry, Juni 2009.

Nr. 64 Dewenter, Ralf; Jaschinski, Thomas; Wiese, Nadine: Wettbewerbliche Auswirkungen eines nichtneutralen Internets, Juli 2009.

Nr. 65 Dewenter, Ralf; Haucap, Justus; Kuchinke, Björn A.: Das Glück und Unglück von Studierenden aus Ost- und Westdeutschland: Ergebnisse einer Befragung in Ilmenau, Bochum und Hamburg, Oktober 2009.

Nr. 66 Kuchinke, Björn A.; Zerth, Jürgen; Wiese, Nadine: Spatial Competition between Health Care Providers: Effects of Standardization, Oktober 2009. 
Nr. 67 Itzenplitz, Anja; Seifferth-Schmidt, Nicole: Warum Klimakonferenzen scheitern, aber dennoch zum Wohl des Weltklimas kooperiert wird, Juli 2010.

Nr. 68 Kallfaß, Hermann H.: Die Aufmerksamkeit für, die Nutzung der und die Werbung in Medien in Deutschland, November 2010.

Nr. 69 Budzinski, Oliver: Empirische Ex-Post Evaluation von wettbewerbspolitischen Entscheidungen: Methodische Anmerkungen, Januar 2012.

Nr. 70 Budzinski, Oliver: The Institutional Framework for Doing Sports Business: Principles of EU Competition Policy in Sports Markets, January 2012.

Nr. 71 Budzinski, Oliver; Monostori, Katalin: Intellectual Property Rights and the WTO, April 2012.

Nr. 72 Budzinski, Oliver: International Antitrust Institutions, Juli 2012.

Nr. 73 Lindstädt, Nadine; Budzinski, Oliver: Newspaper vs. Online Advertising Is There a Niche for Newspapers in Modern Advertising Markets?

Nr. 74 Budzinski, Oliver; Lindstädt, Nadine: Newspaper and Internet Display Advertising - Co-Existence or Substitution?, Juli 2012b.

Nr. 75 Budzinski, Oliver: Impact Evaluation of Merger Control Decisions, August 2012.

Nr. 76 Budzinski, Oliver; Kuchinke, Björn A.: Deal or No Deal? Consensual Arrangements as an Instrument of European Competition Policy, August 2012.

Nr. 77 Pawlowski, Tim, Budzinski, Oliver: The (Monetary) Value of Competitive Balance for Sport Consumers, Oktober 2012.

Nr. 78 Budzinski, Oliver: Würde eine unabhängige europäische Wettbewerbsbehörde eine bessere Wettbewerbspolitik machen?, November 2012.

Nr. 79 Budzinski, Oliver; Monostori, Katalin; Pannicke, Julia: Der Schutz geistiger Eigentumsrechte in der Welthandelsorganisation - Urheberrechte im TRIPS Abkommen und die digitale Herausforderung, November 2012.

Nr. 80 Beigi, Maryam H. A.; Budzinski, Oliver: On the Use of Event Studies to Evaluate Economic Policy Decisions: A Note of Caution, Dezember 2012.

Nr. 81 Budzinski, Oliver; Beigi, Maryam H. A.: Competition Policy Agendas for Industrializing Countries, Mai 2013. 
Nr. 82 Budzinski, Oliver; Müller, Anika: Finanzregulierung und internationale Wettbewerbsfähigkeit: der Fall Deutsche Bundesliga, Mai 2013.

Nr. 83 Doose, Anna Maria: Methods for Calculating Cartel Damages: A Survey, Dezember 2013.

Nr. 84 Pawlowski, Tim; Budzinski, Oliver: Competitive Balance and Attention Level Effects: Theore-tical Considerations and Preliminary Evidence, März 2014.

Nr. 85 Budzinski, Oliver: The Competition Economics of Financial Fair Play, März 2014.

Nr. 86 Budzinski, Oliver; Szymanski, Stefan: Are Restrictions of Competition by Sports Associations Horizontal or Vertical in Nature?, März, 2014.

Nr. 87 Budzinski, Oliver: Lead Jurisdiction Concepts Towards Rationalizing Multiple Competition Policy Enforcement Procedures, Juni 2014.

Nr. 88 Budzinski, Oliver: Bemerkungen zur ökonomischen Analyse von Sicherheit, August 2014.

Nr. 89 Budzinski, Oliver; Pawlowski, Tim: The Behavioural Economics of Competitive Balance: Implications for League Policy and Championship Management, September 2014.

Nr. 90 Grebel, Thomas; Stuetzer, Michael: Assessment of the Environmental Performance of European Countries over Time: Addressing the Role of Carbon

Nr. 91 Emam, Sherief; Grebel, Thomas: Rising Energy Prices and Advances in Renewable Energy Technologies, July 2014.

Nr. 92 Budzinski, Oliver; Pannicke, Julia: Culturally-Biased Voting in the Eurovision Song Contest: Do National Contests Differ?, December 2014. 